\title{
Frequency and Types of Red Cell Alloantibodies in Pregnant Females
}

${ }^{1}$ Umme Habiba, ${ }^{2}$ Asma Munir, ${ }^{3}$ Naseem Akhter, ${ }^{4}$ Sadaf Waris, ${ }^{5}$ Lubna Humayun,

${ }^{1}$ Aafrinish Amanat

${ }^{1}$ Department of Pathology, Akhter Saeed Medical\& Dental College, Lahore

${ }^{2}$ Department of Pathology, Services Institute of Medical Sciences, Lahore

${ }^{3}$ Department of Pathology, Multan Medical \& Dental College, Multan

${ }^{4}$ Department of Oral Pathology, Akhter Saeed Medical\& Dental College, Lahore

${ }^{5}$ Department of Pathology, UCMD, University of Lahore

\begin{abstract}
Introduction: Maternal isoimmunization, also called alloimmunization, occurs when immune system of a pregnant female is sensitized to foreign $\mathrm{RBC}$ surface antigen producing immune process. This maternal blood when goes to fetal circulation, causes immune reaction and disease in case of maternal and fetal blood group differences. Aims \& Objectives: To find out the frequency and types of red cell alloantibodies among females presenting to antenatal care and recognize risk factors for alloimmunization. Place and duration of study: This cross-sectional descriptive study was carried out at Antenatal Clinic of Shaikh Zayed Hospital, Lahore from $1^{\text {st }}$ January 2013 to $31^{\text {st }}$ August 2013. Material \& Methods: Pregnant females with at least one previous pregnancy were typed for $\mathrm{ABO}$ and $\mathrm{Rh}$ antigens. They were screened and typed for red cell alloantibodies. Detailed history was taken to explore for the risk factors. SPSS version 20.0 was used for data analysis, frequencies of different alloantibodies, blood groups and risk factors reported as percentages, age and gravidity in mean \pm s.d. Results: Out of 200 enrolled cases, $6(3 \%)$ had alloantibodies. Of the positive cases, anti-D was found in 3(50\%), anti-C in 2(33.33\%) and anti-Kell in 1(16.67\%). Commonest risk factors were history of peripartum hemorrhage and gynecological procedures. In Rh-negative cases, disparity of spouse Rh group was also main factor. Conclusion: The most common culprit antibody for alloimmunization was anti-D followed by anti-Kell and anti-C. Commonest risk factor for alloimmunization was pregnancy related bleeding and gynecological procedures. Large population-based studies are required to assess true magnitude of the problem.
\end{abstract}

Key words: Alloimmunization, Hemolytic Disease of Fetus and Newborn (HDFN), Pregnancy, Red Cell Alloantibodies.

\section{INTRODUCTION}

$\mathrm{M}$ exposure of foreign red cell antigen inherited by fetus from father ${ }^{1,2}$ to antenatal immune system and subsequent sensitization. Sensitization can also occur following blood transfusion. ${ }^{1}$ More than 50 antigens can cause sensitization and alloimmunization. Rhesus is commonest followed by $\mathrm{ABO}$, while Kidd, Kell and Duffy are less common causes. ${ }^{2}$

$\mathrm{Rh}$ alloimmunization and hemolytic disease of fetus and newborn (HDFN) was first described by Levine and later by Dr. Louis. Incidence of $\mathrm{Rh}$ alloimmunization and subsequent HDN was highest among whites i.e. 15-16 \% and lowest among Asians $(<1 \%){ }^{1}$
Most important general risk factor for alloimmunization is repeated blood transfusions. ${ }^{3}$ Other risk factors include age, gravidity/parity and eventful/bad obstetric and gynaecological history. ${ }^{4}$

$\mathrm{ABO}$ incompatibility occurs usually during first pregnancy because previous antigen exposure is not required. $\mathrm{ABO}$ antibodies are usually IgM type and do not cause HDFN because these cannot cross placenta. However ABO related HDFN can take place in group $\mathrm{O}$ mothers with high titers of naturally occurring Ig $\mathrm{G}$ anti A or B. ${ }^{5}$

For all other blood groups causing HDFN, antigenic exposure by transfusion or fetomaternal hemorrhage (FMH) is necessary. Immunization response after FMH depends on its volume, ABO incompatibility, $\mathrm{RhD}$ phenotypes and gender of the fetus. ${ }^{2}$

In case of incompatibility with $\mathrm{Rh}$ and other blood, the primary immune response is weak and the 
antibodies produced are of IgM type which do not cross placenta, hence chances of HDFN are scarce. ${ }^{6}$ In subsequent exposure, IgG are produced that cross placenta and cause HDFN. Chances of HDFN increase in subsequent pregnancies. ${ }^{7}$

IgG antibodies cross placenta and attack the fetal red blood cells causing hemolysis. This leads to increased bilirubin in fetal circulation. Fetal liver is incapable of conjugation, so extra bilirubin is conjugated and excreted by mother. Neonates develop hyperbilirubinemia.

Both mother and the fetus should undergo proper tests for the timely diagnosis of HDFN and its management. All $\mathrm{Rh}$ negative blood group women having $\mathrm{Rh} \mathrm{D}$ positive husbands should be screened for alloimmunization at their booking visit to antenatal care unit. If the pregnant woman is found to be positive for alloimmunization at any point in time, then antibody titer must be done. The fetus should be screened for anemia with the help of Middle Cerebral Artery-Peak Systolic Velocity. ${ }^{8}$

The dose of anti-D after delivery is 1500 IU to all $\mathrm{Rh}$ negative women. Additional dose of anti-D may be required in females with large fetomaternal hemorrhage. ${ }^{9}$

Aim of this study is to find out the frequency and types of red cell alloantibodies among females presenting to antenatal care and associated risk factors.

\section{MATERIAL AND METHODS}

This cross-sectional descriptive study was conducted at Department of Hematology and Blood Bank at Shaikh Zayed Medical Complex, Lahore. 200 pregnant females between $16^{\text {th }}$ to $18^{\text {th }}$ week of gestation and having second and subsequent pregnancies presenting to Antenatal Clinic of Gynecology and Obstetrics Department, SZH were selected for this study. Pregnant females with history of multiple transfusions, autoimmune diseases, autoimmune hemolytic anemia, malignant disease, immunomodulation and immunosuppressive therapy and those women who tested positive for Direct Coombs Test (DAT) were excluded from the study.

After written informed consent, data were collected by interviewing participants of study and evaluating their medical record on predesigned proforma. $5 \mathrm{ml}$ of peripheral venous blood sample was collected under aseptic measures. Clear serum was used for blood grouping, Direct Antiglobulin Test (DAT) and Indirect Antiglobulin Test (IAT). For antibodies screening, three-cell panel and for antibodies detection, extended cell panel by DiaMed was used. Blood grouping and DAT was done on same day while other tests were done later after thawing preserved sera.

\section{Statistical analysis:}

All the data were analyzed using SPSS version 20.0. The frequencies of different alloantibodies, blood groups and risk factors were reported as percentages. Age and gravidity were reported as mean and standard deviation.

\section{RESULTS}

The study was conducted on 200 pregnant women of reproductive age with at least one previous pregnancy. Age of the women included in the study ranged from 20-40 years with a mean of $29.36 \pm 4.18$ years. (Fig-1)

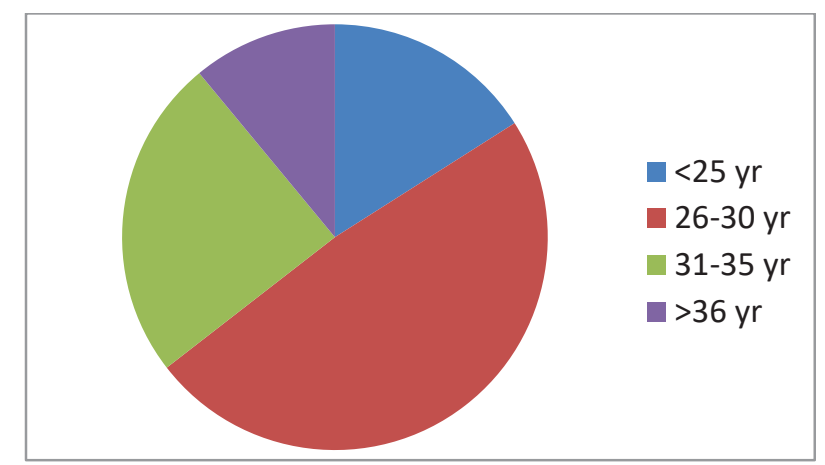

Fig-1: Distribution of women by Age

The gravidity of our cases ranged from 2-7 with a mean of $3.2 \pm 1.8$ and median of 3 .

$23(11.5 \%)$ had no previous live births, 15 (7.5\%) had experienced one or more still births whereas $73(36.5 \%)$ had experienced one or more abortions.

Most common blood group was $\mathrm{B}+\mathrm{ve}$ in $80(40.0 \%)$ women and in $69(34.5 \%)$ of their spouses.

The ABO blood group of 70 women $(35.0 \%)$ and $\mathrm{Rh}$ blood group of $169(84.5 \%)$ was the same as that of their spouses while it was different in rest of them.

History of APH was present in $54(27.0 \%)$ women, IPH in $12(6.0 \%)$ and PPH in $9(4.5 \%)$.

History of gynecological risk factors was present in $18(9.0 \%)$ women and out of them, $16(8.0 \%)$ had previous ectopic pregnancy while $2(1.0 \%)$ had molar pregnancy.

History of obstetrical maneuvers and high risk events was available in 11 (5.5\%) women. External Cephalic Version had been performed in $5(2.5 \%)$, manual removal of placenta in $6(3.0 \%)$, Blunt abdominal trauma in $8(4.0 \%)$ and $2(1.0 \%)$ had a history of manual removal of placenta along with abdominal trauma.

$60(30.0 \%)$ women had the history of gynecological procedures related to previous pregnancies. 
Previous transfusion history was present in 38 $(19.0 \%)$ women. Amongst them $33(16.5 \%)$ had packed red cell transfusion, $2(1.0 \%)$ had platelet transfusion and $3(1.5 \%)$ had been transfused by more than one blood product.

IAT was performed in all cases. $6(3.0 \%)$ women had a positive result showing the presence of antibodies. Red cell alloantibodies were found in all 6 of them. Anti-D was the most common alloantibody and was present in $3(50 \%)$ cases followed by anti-Kell 2(33.3\%) and anti-C 1(16.7\%) Blood group status of alloantibody positive cases is shown in Table-1.

\begin{tabular}{|c|c|c|c|c|c|c|c|c|}
\hline \multirow{1}{*}{} & \multicolumn{7}{|c|}{ Blood Group of Spouse } \\
\cline { 2 - 10 } & & A+ve & B+ve & O+ve & A-ve & B-ve & O-ve & Total \\
\hline \multirow{4}{*}{$\begin{array}{c}\text { Blood } \\
\text { Group } \\
\text { of }\end{array}$} & B+ve & 0 & 0 & 0 & 0 & 0 & 0 & $\mathbf{0}$ \\
\cline { 2 - 10 } Case & O+ve & 1 & 1 & 0 & 0 & 0 & 0 & $\mathbf{2}$ \\
\cline { 2 - 10 } & A-ve & 0 & 1 & 0 & 0 & 0 & 0 & $\mathbf{1}$ \\
\cline { 2 - 10 } & O-ve & 1 & 0 & 0 & 0 & 0 & 0 & $\mathbf{1}$ \\
\cline { 2 - 9 } & Total & $\mathbf{4}$ & $\mathbf{2}$ & $\mathbf{0}$ & $\mathbf{0}$ & $\mathbf{0}$ & $\mathbf{0}$ & $\mathbf{6}$ \\
\hline
\end{tabular}

Table-1: Blood group of women with positive alloantibodies and their spouses

Among these, two were gravida 2, one each was gravida $3,4,6$ and 7 .

Risk factors in previous pregnancies in alloantibody positive cases are shown in Table-2.

\begin{tabular}{|l|c|c|}
\hline Risk factors & N & Percentage \\
\hline Peripartum Bleeding & & \\
\hline APH & 2 & 33.3 \\
\hline IPH & 1 & 16.7 \\
\hline Nil & 3 & 50.0 \\
\hline Total & 6 & 100.0 \\
\hline Gynaecological & & \\
\hline Ectopic & 1 & 16.7 \\
\hline Nil & 5 & 83.3 \\
\hline Total & 6 & 100.0 \\
\hline Obstetrical Risk factors & & \\
\hline Manual Removal of Placenta & 1 & 16.7 \\
\hline Abdominal Trauma & 1 & 16.7 \\
\hline Nil & 4 & 66.7 \\
\hline Total & 6 & 100.0 \\
\hline Procedures & & \\
\hline E\&C & 1 & 16.7 \\
\hline D\&C & 2 & 33.3 \\
\hline Nil & 3 & 50.0 \\
\hline Total & 6 & 100.0 \\
\hline Transfusion History & & \\
\hline Packed RBCs & 2 & 33.3 \\
\hline Nil & 4 & 66.7 \\
\hline Total & 6 & 100.0 \\
\hline
\end{tabular}

Table-2: Risk Factors in Previous Pregnancies in Alloantibody Positive Cases
Summary of alloantibody positive cases is shown in Table-3.

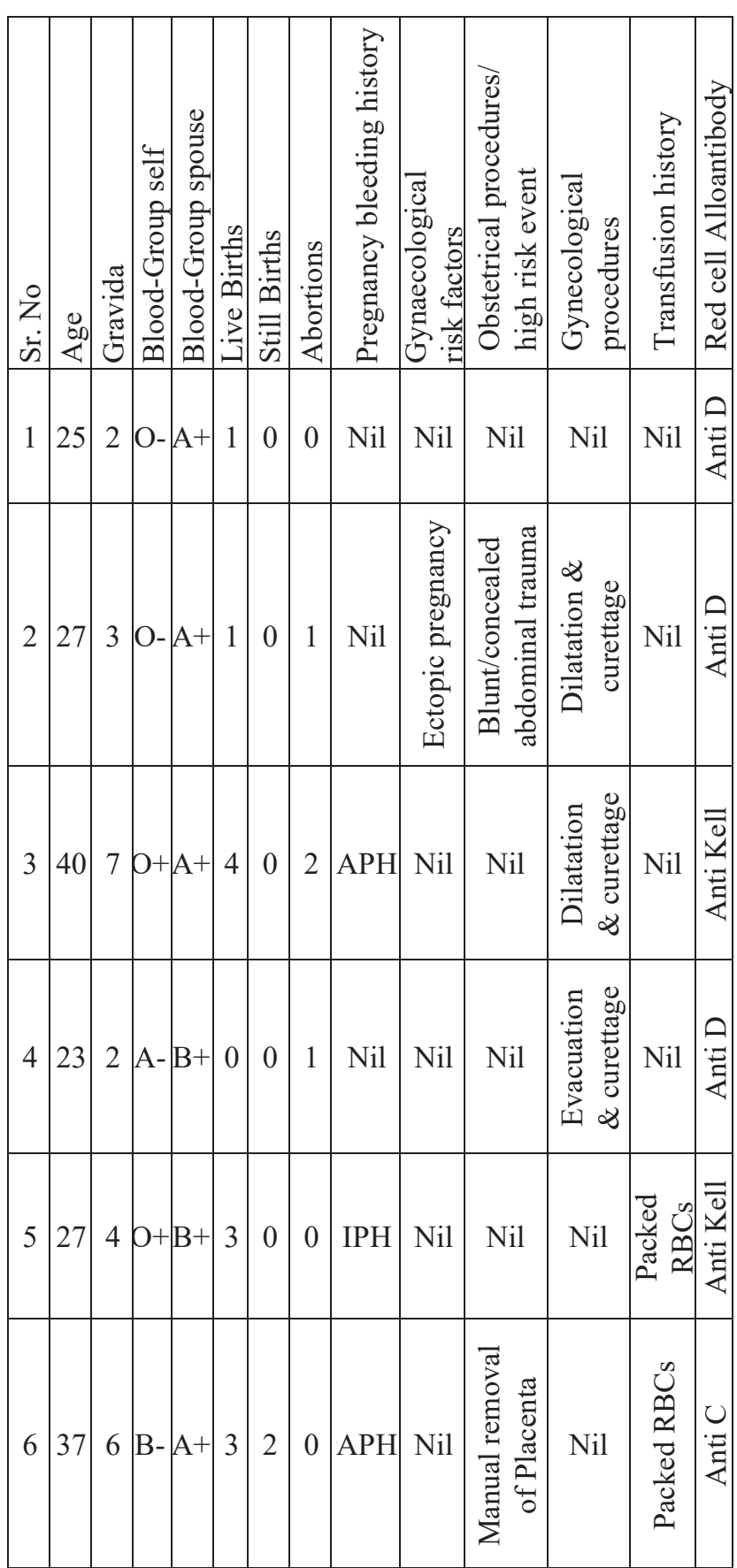

Table-3: Cumulative status of alloantibody positive cases

\section{DISCUSSION}

In the present study, the frequency of red cell alloantibodies and risk factors for alloimmunization were determined in the pregnant women visiting antenatal clinic of Shaikh Zayed Hospital, Lahore. Most severe form of alloimmunization is against Rh-D in which IgG antibodies develop due to sensitization to D antigen in D-negative women. In the developed countries, its incidence is low due to 
anti-D prophylaxis, but in developing countries like Pakistan, there is a significant risk of $\mathrm{Rh}$ incompatibility due to lack of anti-D prophylaxis..$^{10,11}$

The frequency of red cell alloimmunization in pregnant women in the current study is found to be $3 \%$. The frequency studies of red cell alloimmunization from different parts of the world have reported variable results in different countries, regions and centers.

The frequency reported in an Indian study conducted in Delhi was $1.25 \%$. However it was $0.5 \%$ in Sweden and $2.7 \%$ in Netherlands. ${ }^{10,11} \mathrm{~A}$ frequency of $1.92 \%$ was reported from large scale study in Saudi Arabia whereas it was $10.2 \%$ in Mexico and 3.4\% in Nigeria. ${ }^{10,12}$

These variations in frequencies may possibly be due to difference in racial, environmental, socioeconomic status as well as status of anti-D prophylaxis. The frequency of red cell alloantibodies is low in developed countries because of good antenatal services and regular anti-D prophylaxis.

With the advent of anti-D prophylaxis, incidence of HDFN due to ABO and other red cell alloantibodies has relatively increased internationally. ${ }^{13}$

Antenatal services in Pakistan are not equivalent in various regions of the country. Limited research has been done in this field and there are no local guidelines.

In our study, anti-D was detected as the commonest alloantibody among the positive cases. The next frequent alloantibody was anti-Kell followed by anti-C.

In Sweden, anti-Rh were reported as most frequent type of alloantibodies followed by antibodies against Lewis, MNS and Kell system antigens. ${ }^{11} \mathrm{~A}$ study conducted in central province of Saudi Arabia also showed anti-Rh(anti-D being most frequent) to be most common followed by anti-Kell, anti-Kidd, anti-Lewis and anti-Duffy ${ }^{12}$. Similarly, in India, anti-D was reported to be the most common alloantibody, other less frequent being anti-C, anti$\mathrm{M}$ and anti-S. ${ }^{14}$

The results of our study are comparable with most international studies, where anti-D is the most frequent type of alloantibody, other less frequent types being anti-Kell and anti-C. Our results are also comparable to two local studies of Pakistan. The study conducted in Rawalpindi General Hospital showed similar results to our study where anti-D was reported to be most frequent in pregnant females , second most frequent being anti-Kell. ${ }^{15}$ Anti-C was found to be least common among the positive cases in the present study. Our results are therefore consistent with another study conducted in Karachi which also reports anti-C to be less frequent than anti-D. ${ }^{16}$

The most common risk factors for alloimmunization in our study was pregnancy related bleeding in $3(50.0 \%)$ and gynaecological procedures in $3(50.0 \%)$, the next most common risk factor being blood transfusion in $2(33.3 \%)$ and $2(33.3 \%)$ gave history of eventful pregnancies whereas $2(33.3 \%)$ had history of disparity in Rh blood group with husbands. Risk factors were overlapping among the cases. The results were comparable with a study carried out in Western Uganda where history of peripartum hemorrhage was significantly associated with alloimmunization. ${ }^{4}$

Another bicentric scientific study in India showed transfusions and bad obstetrical history playing a remarkable role in alloimmunization. ${ }^{17}$

\section{CONCLUSION}

Shaikh Zayed Hospital is a tertiary care hospital, located amidst the effluent localities of Lahore. Hence it is likely to be serving relatively higher socioeconomic group of patients in comparison to the other public institutions. So results of our study cannot be extrapolated on overall population of Lahore or on the country as a whole. Therefore large scale and multicenter studies need to be conducted in various regions of the country to assess the magnitude of the problem and to observe its pattern. In light of results of the present study, the alloantibody screening should be adopted as a routine antenatal parameter for all the pregnant females and should not be restricted to the mothers with Rh-D negative blood group married to Rh-D positive spouses. This will help in prevention and early management of hemolytic disease of fetus and newborn.

\section{REFERENCES}

1. Arraut A. Erythrocyte alloimmunization and pregnancy. [web].2011[updated Dec 2015; cited 2017 Jan 25]. Available from: https:/emedicine.medscape.com/article/273995overview

2. Cosmi EV, Marzano S, Pizzulo S, Monaco V. Rh-alloimmunization in pregnancy. p177. In: Carrera JM. Recommendations and guidelines for perinatal medicine. Barcelona: Matres Mundi; 2007.

3. Eccles S, Crispin P, Vanniasinkam T. Risk factors for alloimmunization in general patient population. Trans ASci 2015; 52(1): 60-64. 
4. Mbalibulha Y, Muwanguzi E, Mugyenyi G, Natukunda B. Occurrence of anti-D alloantibodies among pregnant women in Kasese District, Western Uganda. JBlood Med 2015; 6:125-129.

5. Firouzi M, Yazdanmehr R, Elyasi H, Birjandi M, Goudarzi A, Almasian M, et al. The prevalence of the $\mathrm{ABO}$ hemolytic disease of the newborn and its complications. Iran J PedHematolOncol. 2018; 8(1):37-47.

6. Salem L, Singer KR. Rh Incompatibility [web]. 2009 [updated 2009 Nov 4, cited 2017 August 5]. Available from:

http://emedicine.medscape.com/article/797150o verview\#showall.

7. Bennardello F, Coluzzi S, Curciarello G, Todros $\mathrm{T}$, Villa S. Recommendations for the prevention and treatment of hemolytic disease of the foetus and newborn. Blood Transfus.2015;13(1):109-34

8. Delaney M, Matthews DC. Hemolytic disease of the fetus and newborn: managing the mother, fetus, and newborn. Am SocHematol Educ. 2015; 146-151.

9. Qureshi H, Massey E, Kirwan D, Davies T, Robson S, White J, Jones J, Allard S. BCSH guideline for the use of anti-D immunoglobulin for the prevention of haemolytic disease of the fetus and newborn. Transfusion Medicine. 2014; 24:8-20.

10. Jeremiah ZA, Mordi A, Buseri FI, AdiasTc. Frequencies of maternal red cell alloantibodies in Port Harcourt, Nigeria. Asian J TransfSci 2011; 5:39-41.

11. Lee BK, Ploner A, Zhang Z, Gryfelt G, Wikman A, Reilly M. Constructing a population-based database from routine maternal screening records: A resource for studying alloimmunization in pregnant women. PLOSONE. 2011; 6(11):1-8.

12. Al-Ibrahim NA, AlSaeed AH. Red blood cell alloimmunization among Saudi pregnant women in the central province of Saudi Arabia. Kuwait Med J 2008; 40:116-123.

13. Basu S, Kaur R, Kaur G. Hemolytic disease of the fetus and newborn: current trends and perspectives. Asian J Transfus Sci.2011;5(1):3-7

14. Pahuja S, Gupta SK, Pujani M, Jain M. the prevalence of irregular erythrocyte antibodies among antenatal women in Delhi. Blood Transfus 2011; 9:388-393.
15. Anwar M, Ali N, Khattak MF, Raashid Y, Karamat KA. A case for comprehensive antenatal screening for blood group antibodies. J Pak Med Assos 1999; 49(10):246-8.

16. Imran M. Frequency of red cell antibodies during pregnancy. Paper presented at: 14th National Hematology Conference; 2012 Feb 2325; Lahore, Pakistan.

17. AgrawalA, Mathur A, Dontula S, Jagannathan L. Red Blood Cell Alloimmunization in Multitransfused Patients: A Bicentric Study in India. G J Trans Med. 2016; 1(1):12-5.

\section{The Authors:}

Dr. Umme Habiba

Assistant Professor Hematology,

Department of Pathology,

Akhter Saeed Medical\& Dental College, Lahore.

Dr. Asma Munir

Assistant Professor Hematology,

Department of Pathology,

Services Institute of Medical Sciences, Lahore.

Dr. Naseem Akhter

Assistant Professor Hematology,

Department of Pathology,

Multan Medical \& Dental College, Lahore.

Dr. Sadaf Waris

Assistant Professor,

Department of Oral Pathology,

Akhter Saeed Medical \& Dental College, Lahore.

Dr. Lubna Humayun

Assistant Professor Hematology,

Department of Pathology,

UCMD, University of Lahore.

Dr. Aafrinish Amanat

Assistant Professor Histopathology,

Department of Pathology,

Akhter Saeed Medical \& Dental College, Lahore.

\section{Corresponding Author:}

Dr. Umme Habiba

Assistant Professor Hematology,

Department of Pathology,

Akhter Saeed Medical\& Dental College, Lahore.

E-mail:umme.habibah2009@gmail.com 